
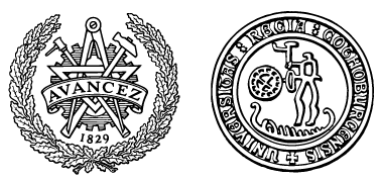

Göteborg ITP preprint

hep-th/0102009

February 2, 2001

\title{
The structure of maximally supersymmetric Yang-Mills theory: constraining higher-order corrections
}

\author{
Martin Cederwall, Bengt E.W. Nilsson and Dimitrios Tsimpis \\ Department of Theoretical Physics \\ Göteborg University and Chalmers University of Technology \\ SE-412 96 Göteborg, Sweden
}

\begin{abstract}
We solve the superspace Bianchi identities for ten-dimensional supersymmetric Yang-Mills theory without imposing any kind of constraints apart from the standard conventional one. In this way we obtain a set of algebraic conditions on certain fields which in the on-shell theory are constructed as composite ones out of the physical fields. These conditions must hence be satisfied by any kind of theory in ten dimensions invariant under supersymmetry and some, abelian or non-abelian, gauge symmetry. Deformations of the ordinary SYM theory (as well as the fields) are identified as elements of a certain spinorial cohomology, giving control over field redefinitions and the distinction between physically relevant higher-order corrections and those removable by field redefinitions. The conditions derived severely constrain theories involving $F^{2}$-level terms plus higher-order corrections, as for instance those derived from open strings as effective gauge theories on D-branes.
\end{abstract}

\footnotetext{
martin. cederwall@fy . chalmers. se

bengt.nilsson@fy.chalmers.se

tsimpis@fy.chalmers.se
} 
$2 \ldots . . \ldots$ Cederwall, Nilsson, Tsimpis: "The structure of maximally supersymmetric..."

\section{INTRODUCTION}

Gauge and reparametrisation invariant theories arise as effective field theories in string theory. In the case of open bosonic strings these field theories correspond to ordinary $F^{2}$ Yang-Mills theory only in the limit of weak fields. In more general situations, due to the appearence of the dimensionful parameter $\alpha^{\prime}$, one typically finds an infinite set of higherorder terms involving arbitrary powers of the Yang-Mills field strength as well as field strengths with any number of covariant derivatives acting on them. To elucidate the structure of these effective actions has turned out to be extremely difficult, and it is only in the abelian case and for constant field strengths that we have any kind of understanding of the complete structure of the action. Under precisely these conditions, the open bosonic string theory is known to generate the Born-Infeld lagrangian

$$
L=-\sqrt{-\operatorname{det}\left(\eta_{a b}+2 \pi \alpha^{\prime} F_{a b}\right)} .
$$

For a quite comprehensive review of the role of the Born-Infeld action in string theory including a large number of references, see ref. [1]. The supersymmetric version of this lagrangian is also known and takes the form

$$
L=-\sqrt{-\operatorname{det}\left(\eta_{a b}+2 \pi \alpha^{\prime} F_{a b}-2\left(2 \pi \alpha^{\prime}\right)^{2} \bar{\lambda} \Gamma_{a} \partial_{b} \lambda+\left(2 \pi \alpha^{\prime}\right)^{4} \bar{\lambda} \Gamma^{c} \partial_{a} \lambda \bar{\lambda} \Gamma_{c} \partial_{b} \lambda\right)},
$$

as can be seen [2] by setting $p=9$ in the action obtained by gauge fixing the reparametrisation invariance and $\kappa$-symmetry of the actions for the $D_{p}$-branes derived in [3].

Adding non-abelian Chan-Paton factors to the ends of the open strings makes it possible to derive non-abelian versions of the effective actions. The importance of these actions have been highlighted recently in connection with the solitonic D-brane solutions in string theory. As explained by Witten in ref. [4], the non-abelian nature of the gauge theory can in this context be understood as arising from a stack of branes by a detailed analysis of all possible configurations with the two ends of the strings ending on different branes in the stack.

Unfortunately, in the non-abelian case our knowledge about the effective action is at a very rudimentary level and there is as yet no situation in which we understand its structure to general order in the fields. In fact there are only partial results up to order $F^{6}$ $[1,5]$ beyond which we have not been able to obtain any information. In the context of the superstring the situation is basically the same. However, here one may pose interesting questions about to what extent $\kappa$-symmetry, extra non-linear supersymmetries, and/or maximal linear supersymmetry constrain the form of higher-order corrections to the ordinary $F^{2}$ super-Yang-Mills theory. In the abelian case $\kappa$-symmetry [3] and non-linear supersymmetries 
[6] are known to be intimately connected to the structure of the Born-Infeld theory. Finding non-abelian generalisations of these arguments has however turned out to be a problem related to the difficult issues that arise when trying to define geometry on a non-commutative (curved) spacetime.

It was found some time ago [7] that at order $F^{4}$, assuming a symmetrised trace over the gauge generators, henceforth denoted $S t r$, and starting from the $S t r F^{4}$ term, supersymmetry is enough to unambiguously produce the structure of the non-abelian action. This is in complete agreement with Tseytlin's suggestion [8] that in the non-abelian case it might suffice to consider the Born-Infeld lagrangian in (1.1) but with all fields in the adjoint of the nonabelian gauge group and with a totally symmetric trace over group generators to eliminate the ordering ambiguities. However, there are strong indications that the Str prescription does not provide the full structure as obtained from string theory at order $F^{6}$ and higher $[9,5]$. Recently Bergshoeff et al. [10] have found, based on an attempt to implement $\kappa$-symmetry in a non-abelian setting, non-Str terms involving fermions (but no pure $F$-terms) also at lower order than this. (The order discussed here is most easily kept track of by giving the fields $F_{\mu \nu}, \lambda_{\alpha}$ and spacetime derivatives canonical four-dimensional mass dimensions despite the fact that we will be dealing exclusively with ten-dimensional super-Yang-Mills theory. When deriving the action from string theory these terms get accompanied by appropriate powers of $\alpha^{\prime}$ which has dimension (mass $)^{-2}$.)

In this paper we will consider ten-dimensional super-Yang-Mills theory [11] and approach the problem of finding the constraints on the possible higher-order corrections implied by (maximal) supersymmetry by embedding the theory in superspace. The theory is then given in terms of a superfield gauge potential $A_{A}=\left(A_{a}, A_{\alpha}\right)$, where the indices $a$ and $\alpha$ refer to the vector and spinor representations, respectively. The corresponding field strength satisfies ordinary superspace Bianchi identities. As we will see in the next section, from these identities one can easily derive the field equations corresponding to the lowest order, i.e., $F^{2}$, theory, supersymmetry transformation rules etc. By relaxing the constraint that leads to these field equations one instead obtains a system of superspace equations that any theory consistent with supersymmetry and gauge invariance must satisfy. In section 3 , we proceed to solve these superspace equations. The solution is in the form of a number of algebraic conditions on the various component fields appearing at different levels in the superfields in terms of which the theory is defined. Section 4 is devoted to a formal development of what we might call spinorial cohomology, where component fields and deformations are obtained as elements of cohomology classes under a fermionic exterior derivative. The understanding obtained elucidates the specific properties enjoyed by maximally supersymmetric Yang-Mills being an on-shell superspace theory and gives a solid underpinning of the approach adopted for finding physically inequivalent deformations. Section 5 is devoted to some comments concerning how this setup might be used to obtain information about pos- 
$4 \ldots \ldots \ldots$ Cederwall, Nilsson, Tsimpis: "The structure of maximally supersymmetric..."

sible higher-order corrections. Some of these comments are of relevance for D-brane physics in string theory and the search for a non-abelian Born-Infeld theory. A more comprehensive discussion containing also explicit expressions at the next, i.e. $F^{4}$, level of corrections will be published elsewhere [12].

\section{IMPLEMENTATION OF GAUGE INVARIANCE IN SUPERSPACE}

This section provides a recapitulation of the superspace techniques in the context of abelian and non-abelian gauge theories, restricted to ten dimensions which corresponds to maximal supersymmetry. We will first set up our conventions and then proceed to derive the equations of motion at order $F^{2}$ by imposing constraints on the superspace Yang-Mills field stength $[13]$.

If we turn a spacetime vector potential $A_{m}(x)$ into a superfield, we obtain the basic object, $A_{M}(x, \theta)$, from which one may construct gauge theories that are manifestly supersymmetric. Here the index $M$ refers to the pair of curved indices $m, \mu$, the first enumerating the ten bosonic components and the second the sixteen fermionic ones. A superspace oneform is constructed by contracting the superspace vector potential with $d x^{m}$ and $d \theta^{\mu}$, which obey opposite statistics to the coordinates $x^{m}$ and $\theta^{\mu}$. From the abelian one-form potential $A$, which transforms under gauge transformations as $\delta A=d \Phi$ where $\Phi$ is a scalar superfield and $d=d x^{m} \partial_{m}+d \theta^{\mu} \partial_{\mu}$, we construct the gauge invariant superfield strength $F=d A$. The corresponding Bianchi identity reads $d F=0$. In the non-abelian case we must of course use the covariant derivative $D=d+A$ instead.

So far no on-shell information has been fed into the equations; in fact any supersymmetric gauge theory must satisfy the superspace Bianchi identity (BI) $D F=0$. When analysing the superspace $\mathrm{BI}$ one usually considers its component equations in tangent space. The reason for this is that since the tangent space structure group is the ordinary Lorentz group $\operatorname{Spin}(1,9)$ and not a supergroup, the vector index $a$ and chiral spinor index ${ }^{*} \alpha$ never mix. Hence the components $F_{a b}, F_{a \beta}$ and $F_{\alpha \beta}$ can be treated as independent and, e.g., constraining a subset of them will not have any effect on the manifest supersymmetry. Reading off the superspace torsion from the supersymmetry algebra we find that superspace always has a non-trivial torsion component, namely $T_{\alpha \beta}^{c}$. In the case of flat superspace considered here,

$$
T_{\alpha \beta}^{c}=2 \Gamma_{\alpha \beta}^{c}
$$

\footnotetext{
* We use upper and lower spinor indices to distinguish the two chiralities, and $16 \times 16 \Gamma$-matrices $\Gamma^{a}{ }_{\alpha \beta}$,
} $\Gamma^{a \alpha \beta}$ (strictly speaking the $\Gamma$-matrices are $32 \times 32$ with these as off-diagonal blocks). 
is the only non-zero one.

In this case, the component form of the superspace BI becomes

$$
\begin{aligned}
D_{(\alpha} F_{\beta \gamma)}+2 \Gamma_{(\alpha \beta}^{c} F_{|c| \gamma)} & =0 \\
2 D_{(\alpha} F_{\beta) c}+D_{c} F_{\alpha \beta}+2 \Gamma_{\alpha \beta}^{d} F_{d c} & =0 \\
D_{\alpha} F_{b c}+2 D_{[b} F_{c] \alpha} & =0 \\
D_{[a} F_{b c]} & =0 .
\end{aligned}
$$

As is well-known the lowest order ten-dimensional supersymmetric Yang-Mills theory is obtained by choosing the constraint [13]

$$
F_{\alpha \beta}=0
$$

The vector part of this constraint, $\left(\Gamma^{a}\right)^{\alpha \beta} F_{\alpha \beta}=0$, is a so called conventional constraint [14] which must be imposed in order to eliminate an unwanted extra vector potential appearing at the first $\theta$ level in $A_{\alpha}$. However, this can always be done without affecting the supersymmetry since it just amounts to a shift of the superfield $A_{\alpha}$ by the vector part of $F_{\alpha \beta}$. To see how the equations of motion emerge, we insert the constraint (2.3) into the above component equations (2.2) leading to the following set of equations:

$$
\begin{aligned}
\Gamma_{(\alpha \beta}^{c} F_{|c| \gamma)} & =0, \\
D_{(\alpha} F_{\beta) c}+\Gamma_{\alpha \beta}^{d} F_{|d| c} & =0, \\
D_{\alpha} F_{b c}+2 D_{[b} F_{c] \alpha} & =0, \\
D_{[a} F_{b c]} & =0 .
\end{aligned}
$$

Instead of just presenting the solution to these equations, we will here take the opportunity to elaborate, in a rather simple situation, on the different steps needed to obtain it. The same procedure will be followed also in the next section where the equations as well as the process of finding the solution is significantly more complicated.

It is convenient to first analyze in detail the representation content of the equations as well as of the fields. Then the goal is to derive all the algebraic relations between the irreducible fields that are hidden in these equations. Decomposing into irreducible repre- 
$6 \ldots . . \ldots$ Cederwall, Nilsson, Tsimpis: "The structure of maximally supersymmetric..."

sentations the symmetric product of three spinor representations we find ${ }^{*} \otimes_{s}^{3}(00010)=$ $(00030) \oplus(10010)$. Thus the first Bianchi identity above can only produce restrictions on fields transforming in the two representations (00030) and (10010). Decomposing also $F_{a \beta}$ we find $(00001) \oplus(10010)$ which we write as

$$
F_{a \beta}=\tilde{F}_{a \beta}+\Gamma_{a \beta \gamma} \lambda^{\gamma},
$$

where $\tilde{F}_{a \beta}$ is $\Gamma$-traceless, $\Gamma^{a \alpha \beta} \tilde{F}_{a \beta}=0 . \lambda$ will turn out to be the physical on-shell spinor field. Comparing the equation content to the field content we see immediately that no information can be obtained about the spinor $\lambda$ while there is one equation for the field in representation (10010). In fact one finds that this equation puts this field to zero. Thus the $(\alpha \beta \gamma)$ Bianchi identity reduces to

$$
F_{a \beta}=\Gamma_{a \beta \gamma} \lambda^{\gamma}
$$

Turning to the second component Bianchi identity (with index structure $a(\beta \gamma)$ ) we repeat the above steps and obtain the table:

$$
\begin{array}{lllllllllll}
D_{\beta} \lambda^{\gamma} & : & (00000) & \oplus & (00011) & \oplus & (01000) & & & & \\
F_{b a} & : & & & & & & (01000) \\
\text { BI } & : & (00000) & \oplus & (00011) & \oplus & (01000) & \oplus & (10020) & \oplus & (20000)
\end{array}
$$

Here the explicit form of the field content at first order in the $\theta$ expansion of $\lambda$ has been written in the equivalent form of a covariant derivative on $\lambda^{\alpha}$ as

$$
D_{\alpha} \lambda^{\beta}=\delta_{\alpha}{ }^{\beta} \Lambda+\frac{1}{2} \Gamma_{\alpha}^{a b}{ }_{\alpha}^{\beta} \Lambda_{a b}+\frac{1}{4 !} \Gamma_{\alpha}^{a b c d}{ }_{\alpha}^{\beta} \Lambda_{a b c d}
$$

The BI yields

$$
\Lambda=0, \quad \Lambda_{a b}=F_{a b}, \quad \Lambda_{a b c d}=0 .
$$

The third BI, of dimension $\frac{5}{2}$, reads

$$
D_{\alpha} F_{a b}=2\left(\Gamma_{[a} D_{b]} \lambda\right)_{\alpha},
$$

\footnotetext{
* We denote irreducible representations of the Lorentz group by highest weight Dynkin labels according to the standard enumeration

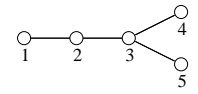


which when inserted into eq. (2.8) together with the solution for the $\Lambda$ 's (2.9) turns into $D_{\alpha} D_{\beta} \lambda^{\gamma}=\Gamma_{\beta}^{a b}{ }_{\beta}^{\gamma}\left(\Gamma_{a} D_{b} \lambda\right)_{\alpha}$. Contraction of this equation with $\Gamma_{c}^{\alpha \beta} \Gamma_{\gamma \delta}^{c}$ gives the equation of motion for the spinor,

$$
\not D \lambda=0 \text {. }
$$

Acting on this equation with a spinor derivative and using eq. (2.8) gives the equation of motion for the vector,

$$
D^{b} F_{a b}-\lambda \Gamma_{a} \lambda=0
$$

where the second term arises from pulling the spinor derivative through the vector derivative and using the dimension- $\frac{3}{2}$ field strength (2.6).

\section{SOlVing the SUPERSPACE BiAnChI IDENTITIES OFF-SHELL}

In this section we relax the constraint $F_{\alpha \beta}=0$ and derive what we will refer to as the off-shell equations. These equations will include dynamical equations for $A_{a}$ and $\lambda_{\alpha}$ which however contain other unspecified auxiliary fields taking the theory off the mass-shell derived from $F_{\alpha \beta}=0$. The effects of relaxing the constraint were discussed in ref. [13], which ruled out the possibility of constructing an off-shell lagrangian.

As discussed in the previous section the constraint $F_{\alpha \beta}=0$ on the dimension-1 field strength puts the theory on the ordinary (lowest order) mass shell. In order to relax it, we set $[13]$

$$
F_{\alpha \beta}=\frac{1}{5 !} \Gamma_{\alpha \beta}^{a_{1} \ldots a_{5}} J_{a_{1} \ldots a_{5}}
$$

where we choose $J$ to be anti-selfdual, i.e., $J \in(00020)$. In principle, there could be a $\Gamma^{(1)}$

term, which is set to zero by a conventional constraint as explained above. At dimension $\frac{3}{2}$, $F$ is expanded as

$$
F_{a \alpha}=\tilde{F}_{a \alpha}+\left(\Gamma_{a} \lambda\right)_{\alpha},
$$

where $\tilde{F} \in(10010)$ is $\Gamma$-traceless, and $\lambda \in(00001)$ ( $\lambda$ is the physical spinor field). At the same time, $D_{\alpha} J_{\text {abcde }}$ is expanded according to $(00010) \otimes(00020)=(00030) \oplus(00110) \oplus(10010)$ :

$$
D J_{a b c d e}=\tilde{J}_{a b c d e}+10 \Gamma_{[a b} \tilde{J}_{c d e]}+5 \Gamma_{[a b c d} \tilde{J}_{e]},
$$

whose different irreducible parts can be reexpressed in terms of covariant derivatives on 
$J_{a b c d e}$ by means of the following inversion formulæ:

$$
\begin{aligned}
\tilde{J}_{a} & =\frac{1}{1680} \Gamma^{b c d e} D J_{b c d e a}, \\
\tilde{J}_{a b c} & =-\frac{1}{12} \Gamma^{d e} D J_{d e a b c}-\frac{1}{224} \Gamma_{[a b} \Gamma^{d e f g} D J_{|d e f g| c]}, \\
\tilde{J}_{a b c d e} & =D J_{a b c d e}+\frac{5}{6} \Gamma_{[a b} \Gamma^{f g} D J_{|f g| c d e]}+\frac{1}{24} \Gamma_{[a b c d} \Gamma^{f g h i} D J_{|f g h i| e]} .
\end{aligned}
$$

The first equation to be solved is the one in $(2.2)$ of dimension $\frac{3}{2}$. It reads

$$
0=D_{(\alpha} F_{\beta \gamma)}+T_{(\alpha \beta}{ }^{a} F_{|a| \gamma)}
$$

and contains the irreps $\otimes_{s}^{3}(00010)=(00030) \oplus(10010)$. Analyzing eq. (3.5) we find that it sets $\tilde{J}_{a b c d e}$ to zero, leaves $\tilde{J}_{a b c}$ and $\lambda$ unconstrained and relates $\tilde{J}_{a}$ and $\tilde{F}_{a}$ in the following way:

$$
\tilde{F}_{a \alpha}=-7 \tilde{J}_{a \alpha} .
$$

The vanishing of the (00030) component is absolutely essential, and has a cohomological interpretation (see the following section). It is the only condition that the superfield $J_{a b c d e}$ has to satisfy. Once it is fulfilled, the modified equations of motion follow.

At dimension 2 the relevant equation reads

$$
\begin{aligned}
0 & =D_{a} F_{\alpha \beta}-2 D_{(\alpha} F_{|a| \beta)}+T_{\alpha \beta}{ }^{b} F_{b a} \\
& =\frac{1}{5 !} \Gamma_{\alpha \beta}^{a_{1} \ldots a_{5}} D_{a} J_{a_{1} \ldots a_{5}}+14 D_{(\alpha} \tilde{J}_{|a| \beta)}-2 \Gamma_{a(\alpha|\gamma|} D_{\beta)} \lambda^{\gamma}+2 \Gamma_{\alpha \beta}{ }^{b} F_{b a} .
\end{aligned}
$$

The irreducible content of the various quantities is

$$
\begin{array}{llllllllllll}
D_{a} J_{a_{1} \ldots a_{5}} & : & & & (00011) & & & \oplus & (10020) & & \\
D_{(\alpha} \tilde{J}_{|a| \beta)} & : & & & & (00011) & \oplus & (01000) & \oplus & (10020) & & \\
D_{\beta} \lambda^{\gamma} & : & (00000) & \oplus & (00011) & \oplus & (01000) & & & & \\
F_{b a} & : & & & & & (01000) & & & & \\
\mathrm{BI} & : & (00000) & \oplus & (00011) & \oplus & (01000) & \oplus & (10020) & \oplus & (20000)
\end{array}
$$

(in the second row, symmetrisation $(\alpha \beta)$ has been used, as well as the property $\tilde{J} \sim D J$, which takes away (20000)). Schematically, the equations are

$$
\begin{aligned}
& \text { (00000) : } D_{\alpha} \lambda^{\alpha}=0 \\
& (00011): D^{2} J+\left(D \Gamma^{(4)} \lambda\right) \sim 0, \\
& (01000): D^{2} J+\left(D \Gamma^{(2)} \lambda\right)+F \sim 0, \\
& \text { (10020) : } D^{2} J \sim 0 \text {. }
\end{aligned}
$$


At the second level in $J$, one has the irreps $(00011) \oplus(01000) \oplus(10020) \oplus(00120) \oplus(01011) \oplus$ (10100), of which only the first three take part in the BI. We write

$$
\begin{aligned}
D_{[\alpha} D_{\beta]} J_{a b c d e}=10 & \Gamma_{[a b c} K_{d e]}+\frac{1}{2} \Gamma_{a b c d e}{ }^{f g} K_{f g} \\
& +10 \Gamma_{[a b}^{f} K_{|f| c d e]}+\frac{5}{6} \Gamma_{[a b c d}^{f g h} K_{|f g h| e]} \\
& +\frac{5}{2}\left(\Gamma_{[a}^{g h}\right)_{\alpha \beta} S_{|g h| b c d, e]}+\ldots, \\
D_{(\alpha} D_{\beta)} J_{a b c d e}=- & \frac{1}{2} T_{\alpha \beta}{ }^{f} D_{f} J_{a b c d e}-\frac{1}{2}\left[F_{\alpha \beta}, J_{a b c d e}\right] \\
=- & \Gamma_{\alpha \beta}^{f} D_{f} J_{a b c d e}-\frac{1}{2 \cdot 5 !} \Gamma_{\alpha \beta}^{f g h i j}\left[J_{f g h i j}, J_{a b c d e}\right]
\end{aligned}
$$

(the $[J, J]$ part only contains the representations $(00120) \oplus(10100)$ which do not enter the $\operatorname{dim} 2 \mathrm{BI})$. The inversions for the $K$ 's will be used later and read

$$
\begin{aligned}
K_{a b} & =\frac{1}{5376}\left(D \Gamma^{c d e} D\right) J_{c d e a b}, \\
K_{a b c d} & =\frac{1}{480}\left(D \Gamma_{[a}^{f g} D\right) J_{|f g| b c d]} .
\end{aligned}
$$

We also decompose $D \lambda$ as

$$
D_{\alpha} \lambda^{\beta}=\delta_{\alpha}^{\beta} \Lambda+\frac{1}{2} \Gamma_{\alpha}^{a b}{ }_{\alpha}^{\beta} \Lambda_{a b}+\frac{1}{4 !} \Gamma_{\alpha}^{a b c d}{ }_{\alpha}^{\beta} \Lambda_{a b c d} .
$$

The equations become:

$$
\begin{array}{ll}
(00000): & 0=\Lambda \\
(00011): & 0=\frac{7}{30} D^{f} J_{f a b c d}+2 K_{a b c d}-\Lambda_{a b c d} \\
(01000): & 0=\frac{28}{5} K_{a b}+\Lambda_{a b}-F_{a b}, \\
(10020): & 0=S_{a b c d e, f}+\frac{5}{6}\left(D_{f} J_{a b c d e}+D_{[a} J_{b c d e] f}-\eta_{f[a} D^{g} J_{|g| b c d e]}\right) .
\end{array}
$$

There is one important consistency check here: when $\tilde{J}_{a b c d e}$ in the representation (00030) vanishes at dimension $\frac{3}{2}$, there is no new independent (10020) at dimension 2 (see figure 1). The equation obtained from acting with one spinor derivative on $\tilde{J}_{a b c d e}=0$ will contain a part in (10020) that had better coincide with the one in eq. (3.13), if we are not to get a differential constraint on $J$. We have checked that this is the case. Similar consistency conditions arise at higher dimensions, and are always automatically fulfilled once the (00030) representation at dimension $\frac{3}{2}$ vanishes. The (00030) constraint also implies that the (00120) part of $D_{[\alpha} D_{\beta]} J_{a b c d e}$ is expressible in terms of the one in $[J, J]$. The $(10100) \oplus(01011)$ part remains unconstrained. 
The dimension- $\frac{5}{2}$ Bianchi identity reads

$$
0=2 D_{[a} F_{b] \alpha}+D_{\alpha} F_{a b}=-14 D_{[a} \tilde{J}_{b] \alpha}-2 \Gamma_{[a|\alpha \beta|} D_{b]} \lambda^{\beta}+D_{\alpha} F_{a b}
$$

Inserting this expression for $D_{\alpha} F_{a b}$ into one spinor derivative on $D_{\alpha} \lambda^{\beta}$ from above gives

$$
\begin{array}{r}
D_{\alpha} D_{\beta} \lambda^{\gamma}=\frac{1}{2} \Gamma_{\beta}^{a b}{ }_{\beta}^{\gamma}\left[2\left(\Gamma_{a} D_{b} \lambda\right)_{\alpha}+14 D_{a} \tilde{J}_{b \alpha}-\frac{28}{5} D_{\alpha} K_{a b}\right] \\
+\frac{1}{24} \Gamma^{a b c d}{ }_{\beta}^{\gamma}\left[\frac{7}{30} D_{\alpha} D^{f} J_{f a b c d}+2 D_{\alpha} K_{a b c d}\right]
\end{array}
$$

and contracting with $\Gamma_{g}^{\alpha \beta} \Gamma^{g} \delta \gamma$ gives (a preliminary form of) the equation of motion for $\lambda$ :

$$
0=\not D \lambda+\frac{3}{5} D^{a} \tilde{J}_{a}+\frac{1}{3600} \Gamma^{a b c d} D D^{f} J_{f a b c d}+\frac{6}{25} \Gamma^{a b} D K_{a b}+\frac{1}{420} \Gamma^{a b c d} D K_{a b c d}
$$

In order to simplify the equation of motion (3.16) for $\lambda$ we need to expand the last three terms. As seen in figure 1, there is one spinor (00010) at dimension $\frac{5}{2}$ in $J$. We parametrise it as

$$
D_{[\alpha} D_{\beta} D_{\gamma]} J^{a b c d e}=30 \Gamma_{[\alpha \beta}^{[a b c}\left(\Gamma^{d e]} \psi\right)_{\gamma]}+\ldots
$$

with the inversion

$$
\psi_{\alpha}=-\frac{1}{840 \cdot 3 ! \cdot 5 !} \Gamma_{a b c}{ }^{\beta \gamma} \Gamma_{d e \alpha}{ }^{\delta} D_{[\beta} D_{\gamma} D_{\delta]} J^{a b c d e} .
$$

Since there is no $(00010)$ in $\wedge^{3}(00010) \otimes(00002)$, the right hand side of eq. (3.17) is automatically anti-selfdual. We insert this into one spinor derivative on eq. (3.11) to obtain, after some calculation,

$$
\begin{aligned}
\Gamma^{a b} D K_{a b} & =-\frac{225}{2} \psi+\frac{5}{2} D^{a} \tilde{J}_{a}+\frac{1}{2016} \Gamma^{a b c d e}\left[\lambda, J_{a b c d e}\right], \\
\Gamma^{a b c d} D K_{a b c d} & =-1260 \psi-140 D^{a} \tilde{J}_{a}-\frac{1}{36} \Gamma^{a b c d e}\left[\lambda, J_{a b c d e}\right] .
\end{aligned}
$$

The $\psi$ terms come from the totally antisymmetrised product of three derivatives, while terms with mixed symmetrisation give the $\tilde{J}$ and commutator terms from torsion and curvature, respectively. We use $D_{\delta} D_{[\beta} D_{\gamma]}=D_{[\delta} D_{\beta} D_{\gamma]}+\frac{4}{3} D_{(\delta} D_{\beta)} D_{\gamma}-\frac{2}{3} D_{\beta} D_{(\delta} D_{\gamma)}$, where antisymmetrisation $[\beta \gamma]$ is understood in the right hand side. The equation of motion for $\lambda$ is

$$
0=\not D \lambda-30 \psi+\frac{4}{3} D^{a} \tilde{J}_{a}+\frac{5}{126 \cdot 5 !} \Gamma^{a b c d e}\left[\lambda, J_{a b c d e}\right]
$$

To derive the equation of motion for $F$, we start from the equation of motion for $\lambda$, act with a spinor derivative and contract with a $\Gamma$ matrix, i.e., write eq. (3.20) as $0=\Lambda^{(1)}+$ 
Cederwall, Nilsson, Tsimpis: "The structure of maximally supersymmetric...” ....... 11

$\Lambda^{(2)}+\Lambda^{(3)}+\Lambda^{(4)} \equiv \Lambda$ and consider the equation $0=D \Gamma_{a} \Lambda \equiv L_{a} \equiv L_{a}^{(1)}+L_{a}^{(2)}+L_{a}^{(3)}+L_{a}^{(4)}$. Then,

$$
\begin{aligned}
L_{a}^{(1)}= & 16 D^{b}\left(F_{a b}-\frac{28}{5} K_{a b}\right)-14\left\{\lambda, \tilde{J}_{a}\right\}-16 \lambda \Gamma_{a} \lambda, \\
L_{a}^{(2)}= & 576 w_{a}-\frac{64}{3} D^{b} K_{a b}-\frac{40}{9}\left\{\lambda, \tilde{J}_{a}\right\}-\frac{40}{3} \tilde{J}_{b} \Gamma_{a} \tilde{J}^{b}+\frac{2}{105} \tilde{J}_{b c d} \Gamma_{a} \tilde{J}^{b c d} \\
& +\frac{52}{189}\left[K_{b c d e}, J_{a} b c d e\right]+\frac{2}{567}\left[D^{f} J_{f b c d e}, J_{a}^{b c d e}\right] \\
L_{a}^{(3)}=- & \frac{256}{15} D^{b} K_{a b}+\frac{8}{3}\left\{\lambda, \tilde{J}_{a}\right\}-\frac{56}{3} \tilde{J}_{b} \Gamma_{a} \tilde{J}^{b} \\
L_{a}^{(4)}= & -\frac{50}{9}\left\{\lambda, \tilde{J}_{a}\right\}+\frac{20}{189}\left[K_{b c d e}, J_{a}{ }^{b c d e}\right]+\frac{1}{81}\left[D^{f} J_{f b c d e}, J_{a}^{b c d e}\right] .
\end{aligned}
$$

Here, we have defined the vector $w$ at fourth level in $J$ by

$$
D_{[\alpha} D_{\beta} D_{\gamma} D_{\delta]} J^{a b c d e}=60 \Gamma_{[\alpha \beta}^{[a b c} \Gamma_{\gamma \delta]}^{d e] f} w_{f}+\ldots
$$

with the inversion

$$
w_{a}=\frac{1}{4032 \cdot 4 ! \cdot 5 !} \Gamma_{a b c}^{[\alpha \beta} \Gamma_{d e f}^{\gamma \delta]} D_{\alpha} D_{\beta} D_{\gamma} D_{\delta} J^{b c d e f},
$$

and used $D_{\alpha} D_{[\beta} D_{\gamma} D_{\delta]}=D_{[\alpha} D_{\beta} D_{\gamma} D_{\delta]}+\frac{3}{2} D_{(\alpha} D_{\beta)} D_{\gamma} D_{\delta}-D_{\beta} D_{(\alpha} D_{\gamma)} D_{\delta}+\frac{1}{2} D_{\beta} D_{\gamma} D_{(\alpha} D_{\delta)}$ (antisymmetrisation $[\beta \gamma \delta]$ understood) in $L^{(2)}$.

The equation of motion for $A$ is thus

$$
\begin{aligned}
0= & D^{b} F_{a b}-\lambda \Gamma_{a} \lambda-8 D^{b} K_{a b}+36 w_{a}-\frac{4}{3}\left\{\lambda, \tilde{J}_{a}\right\}-2 \tilde{J}_{b} \Gamma_{a} \tilde{J}^{b}+\frac{1}{140 \cdot 3 !} \tilde{J}_{b c d} \Gamma_{a} \tilde{J}^{b c d} \\
& +\frac{1}{42}\left[K_{b c d e}, J_{a}^{b c d e}\right]+\frac{1}{42 \cdot 4 !}\left[D^{f} J_{f b c d e}, J_{a}{ }^{b c d e}\right]
\end{aligned}
$$

(00020)

$3 / 2:$

(00030) (00110) (10010)

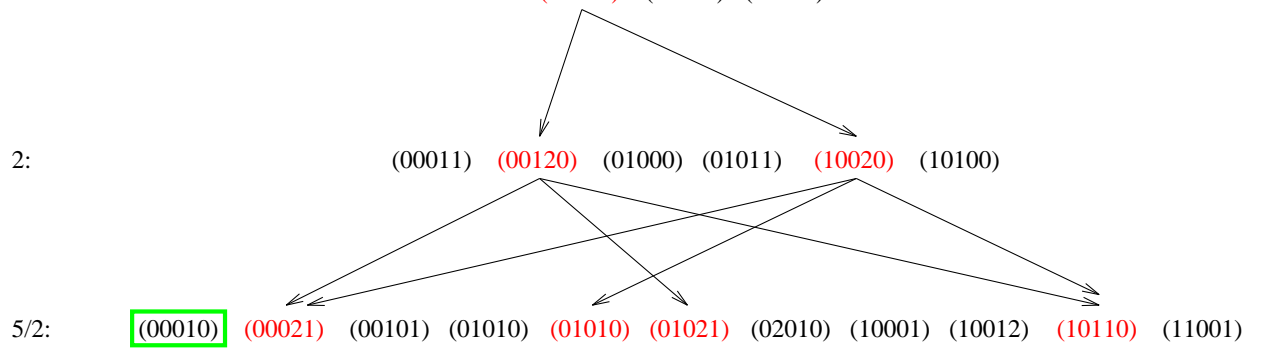

Figure 1. The representations in $J$ up to dimension $\frac{5}{2}$. The arrows show how the (00030) constraint propagates. The boxed representation is the spinor $\psi$ responsible for the right hand 
$12 \ldots . . \ldots$ Cederwall, Nilsson, Tsimpis: "The structure of maximally supersymmetric..."

side of the equation of motion for $\lambda$. The vector $w$ at dimension 3, occurring in the equation of motion for $A$, is also outside the (00030) superfield.

\section{FiELDS AND INTERACTIONS FROM SPINORIAL COHOMOLOGY}

In this section, we would like to present an argument that gives conceptual support to the procedure we have adopted for constructing higher-order corrections to the ordinary super-Yang-Mills theory.

The gauge potentials are $A_{\alpha}$ and $A_{a}$. However, the spinor potential already contains a vector (of correct dimension) at the $\theta$ level, and this is the reason why a conventional constraint is needed in order to have one vector potential. This constraint is

$$
\Gamma_{a}^{\alpha \beta} F_{\alpha \beta}=0
$$

which implies that (in the abelian case, for simplicity)

$$
A_{a}=-\frac{1}{32} D \Gamma_{a} A
$$

The rest of $F_{\alpha \beta}$, which lies in (00020), does not contain $A_{a}$. We have also noted that part of the dimension- $\frac{3}{2}$ Bianchi identity states the vanishing of the (00030) component of $D_{\alpha} F_{\beta \gamma}$.

These observations make it natural to consider, not the sequence of completely symmetric representations in spinor indices, but a restriction of it, namely the sequence of $\operatorname{Spin}(1,9)$ representations

$$
(00000) \stackrel{\Delta_{0}}{\longrightarrow}(00010) \stackrel{\Delta_{1}}{\longrightarrow}(00020) \stackrel{\Delta_{2}}{\longrightarrow} \ldots \stackrel{\Delta_{n-1}}{\longrightarrow}(000 n 0) \stackrel{\Delta_{n}}{\longrightarrow} \ldots
$$

The representation $r_{n} \equiv(000 n 0)$ is the part of the totally symmetric product of $n$ chiral spinors that has vanishing $\Gamma$-trace, and may be represented tensorially as $C_{\alpha_{1} \ldots \alpha_{n}}=$ $C_{\left(\alpha_{1} \ldots \alpha_{n}\right)}, \Gamma_{a}{ }^{\alpha_{1} \alpha_{2}} C_{\alpha_{1} \alpha_{2} \alpha_{3} \ldots \alpha_{n}}=0$. For $n=2, C$ is an anti-selfdual five-form, for $n=3$ a $\Gamma$-traceless anti-selfdual five-form spinor, etc.

The operator $\Delta_{n}: r_{n} \longrightarrow r_{n+1}$ can schematically be written as $\Delta_{n} C_{n}=\Pi\left(r_{n+1}\right) D C_{n}$, where $D$ is the exterior covariant derivative $D=d \theta^{\alpha} D_{\alpha}$ and $\Pi\left(r_{n}\right)$ is the algebraic projection from $\otimes_{s}^{n}(00010)$ to $(000 n 0)$. It is straightforward to write an explicit tensorial form for $\Delta$ by subtracting $\Gamma$-traces from $D C$, but it will not be used here. 
It is straightforward to show that, for an abelian gauge group and standard flat superspace, the sequence (4.3) forms a complex, i.e., that $\Delta^{2}=0$. This follows simply from the fact that while $\left\{D_{\alpha}, D_{\beta}\right\}=-T_{\alpha \beta}{ }^{c} D_{c}$, the torsion only has a component $2 \Gamma_{\alpha \beta}{ }^{c}$ which is projected out by $\Pi\left(r_{n}\right)$.

The question immediately arises whether this complex contains any non-trivial cohomology. It is sometimes stated that fermionic cohomology is trivial. This is true when one considers the complex of symmetric multi-spinors but, as we will see, not when projected on the $r_{n}$ 's.

To investigate the content at each $n$, one has to expand the superfields $C_{\alpha_{1} \ldots \alpha_{n}}$ in irreducible component fields. The representation occurring at level $\ell$ (multiplying $\theta^{\ell}$ ) in $C_{n}$ is given as $r_{n}^{\ell} \equiv \wedge^{\ell}(00010) \otimes(000 n 0)$. We would like to keep track of dimensions of fields, so we give $C_{n}$ mass dimension $\frac{n}{2}$, so that a component of $C_{n}$ at level $\ell$ has dimension $\frac{n+\ell}{2}$. Likewise, the cohomology is decomposed as $\mathscr{H}^{n} \equiv \operatorname{Ker} \Delta_{n} / \operatorname{Im} \Delta_{n-1}=\oplus_{\ell} \mathscr{H}^{n, \ell}$. The cohomology is purely algebraic, and is calculated simply as $\mathscr{H}^{n, \ell}=r_{n}^{\ell} \ominus r_{n-1}^{\ell+1} \ominus r_{n+1}^{\ell-1}$ where the second and third terms represent the level $\ell$ contents of $\operatorname{Im} \Delta_{n-1}$ and $\left(\operatorname{Ker} \Delta_{n}\right)^{\perp}$ respectively, and where " $\ominus$ " means subtraction of an irreducible representation only if it is already present.

To be explicit, we present the calculation of $\mathscr{H}^{1}$. We have

$$
\begin{aligned}
\mathscr{H}^{1,0}= & r_{1}^{0} \ominus r_{0}^{1}=(00010) \ominus(00010)=0, \\
\mathscr{H}^{1,1}= & r_{1}^{1} \ominus r_{0}^{2} \ominus r_{2}^{0} \\
= & (00010) \otimes(00010) \ominus \wedge^{2}(00010) \ominus(00020) \\
= & (10000) \oplus(00100) \oplus(00020) \ominus(00100) \ominus(00020) \\
= & (10000), \\
\mathscr{H}^{1,2}= & r_{1}^{2} \ominus r_{0}^{3} \ominus r_{2}^{1} \\
= & \wedge^{2}(00010) \otimes(00010) \ominus \wedge^{3}(00010) \ominus(00010) \otimes(00020) \\
= & (00110) \oplus(01001) \oplus(10010) \oplus(00001) \\
& \ominus(01001) \ominus[(00030) \oplus(00110) \oplus(10010)] \\
= & (00001), \\
\mathscr{H}^{1,3}= & r_{1}^{3} \ominus r_{0}^{4} \ominus r_{2}^{2} \\
= & \wedge^{3}(00010) \otimes(00010) \ominus \wedge^{4}(00010) \ominus \wedge^{2}(00010) \otimes(00020) \\
= & (00011) \oplus(01000) \oplus(01011) \oplus(02000) \oplus(10002) \oplus(10100) \\
& \ominus[(02000) \oplus(10002)] \\
& \ominus[(00011) \oplus(00120) \oplus(01000) \oplus(01011) \oplus(10020) \oplus(10100)] \\
= & 0
\end{aligned}
$$


$14 \ldots \ldots \ldots$ Cederwall, Nilsson, Tsimpis: "The structure of maximally supersymmetric..."

Higher $\mathscr{H}^{1, \ell}$ vanish. Using the dimensions instead of the level, we find that

$$
\mathscr{H}^{1}=(10000)_{1} \oplus(00001)_{\frac{3}{2}}
$$

The interpretation of this result is clear. $C_{1}$ is the spinor potential $A_{\alpha}$, and the cohomology represents the physical component fields in the vector multiplet, $A_{a}$ of dimension 1 and $\lambda^{\alpha}$ of dimensions $\frac{3}{2}$. Subtraction of $\operatorname{Im} \Delta_{0}$ means counting fields modulo gauge transformations, and subtraction of $\left(\operatorname{Ker} \Delta_{1}\right)^{\perp}$ reflects the constraint $F_{\alpha \beta}=0$. The cohomology is not supersymmetric, since the manipulations so far only involved spinorial derivatives. Imposing the complete Bianchi identities, as shown in section 3 , leads to the equations of motion for the component fields.

Let us continue with the second cohomology $\mathscr{H}^{2}$. The calculation is completely analogous, and the result is that it contains a spinor (00010) at $\ell=3$ and a vector at $\ell=4$ :

$$
\mathscr{H}^{2}=(00010)_{\frac{5}{2}} \oplus(10000)_{3}
$$

These components match the ones of the currents occurring in the equations of motion for $\lambda$ and $A$. We can thus identify a deformation of the theory with a field strength $F_{\alpha \beta}$ being an element of $\mathscr{H}^{2}$. Now, subtraction of $\operatorname{Im} \Delta_{1}$ means counting modulo field redefinitions, and subtraction of $\left(\operatorname{Ker} \Delta_{2}\right)^{\perp}$ is related to the dimension- $\frac{3}{2}$ Bianchi identity, which implies the vanishing of the (00030) part of $D_{\alpha} F_{\beta \gamma}$.

For a non-abelian gauge group, one can imagine starting from the undeformed theory with $F_{\alpha \beta}=0$, and trying to deform it infinitesimally by introducing some non-zero $F_{\alpha \beta}$. Then $\Delta$ is defined with respect to the undeformed theory, and an infinitesimal deformation is an element of the cohomology. Finite deformations demand a more refined analysis taking into account the interplay between terms of different orders in an expansion parameter (e.g. $\left.\alpha^{\prime}\right)$. Field strengths expressible as $F=\Delta a$ can be absorbed into the spinor potential through a field redefinition (this will be used explicitly in section 5 and in a forthcoming publication [12]). We then get information about physically inequivalent deformations of supersymmetric gauge theories.

The statement that (part of) the field strength belongs to a non-trivial cohomology class naïvely seems to contradict the statement that it is obtained from a gauge potential (which we made explicit use of when deriving the field equations from the Bianchi identities). However, when the rest of the Bianchi identities are imposed, they will imply equations of motion. Let us take the spinor equation as example. It reads $\not D \lambda \sim \psi+\ldots$ If the cohomology class is trivial, the right hand side will be expressible as $\not D \mu$, so that the deformation is removed by a field redefinition. If the cohomology class is non-trivial, on the other hand, the 
equation of motion states that the right hand side is $\not D \lambda$, and in this sense the equations of motion resolve the cohomology.

It is instructive to consider the analogous complex for six-dimensional super-Yang-Mills with $N=(1,0)$ supersymmetry [15]. This theory has an off-shell formulation in terms of the vector, the spinor and a triplet of auxiliary scalars of dimension 2 . The complex is

$$
(000)(0) \stackrel{\Delta_{0}}{\longrightarrow}(100)(1) \stackrel{\Delta_{1}}{\longrightarrow}(200)(2) \stackrel{\Delta_{2}}{\longrightarrow} \ldots \stackrel{\Delta_{n-1}}{\longrightarrow}(n 00)(n) \stackrel{\Delta_{n}}{\longrightarrow} \ldots
$$

Indeed, the first cohomology is

$$
\mathscr{H}^{1}=(010)(0)_{1} \oplus(001)(1)_{\frac{3}{2}} \oplus(000)(2)_{2}
$$

where the representations are given as standard Dynkin labels for $\operatorname{Spin}(1,5) \times \mathrm{SU}(2)$ (the second factor being the R-symmetry group). The second cohomology is trivial, which also is expected - setting $F_{\alpha \beta}$ to zero does not put the theory on-shell, and the value of $F_{\alpha \beta}$ does not contain any information about interactions, it can be set to zero by a field redefinition.

Finally, we would like to comment on a potentially interesting observation concerning the ten-dimensional theory. While $\mathscr{H}^{n}, n \geq 4$ seem to vanish, the third cohomology $\mathscr{H}^{3}$ is non-trivial, and contains only a scalar of dimension 4 , which is the dimension of a lagrangian. We do not yet understand what this signifies.

\section{Conclusions and outlook}

In this paper we use superspace techniques to derive a set of algebraic constraints on the irreducible field components in the superfield strengths $F_{A B}$. Since some of these field components will correspond to composite operators in theories with higher-order corrections, the algebraic constraints will provide restrictions on the possible explicit form of the corrections. In particular, the abelian Born-Infeld action must satisfy these restrictions as must its non-abelian kin whose lagrangian we have very limited information about.

Whereas $\kappa$-symmetry and non-linear supersymmetries are known to play a very important role in dictating the form of the abelian Born-Infeld action, it is not known how much of the non-linear structure can be deduced from the maximal linear supersymmetry alone. We will return to this question in a forthcoming publication [12], and conclude this section by briefly discussing the role of $F_{\alpha \beta}$ in this context.

We introduced the five-form $J_{a b c d e}$ in eq. (3.1). As follows from the results in section 3 , expressing $J_{a b c d e}$ in terms of the physical fields $F_{a b}$ and $\lambda_{\alpha}$ will give rise to field 
equations with higher-order corrections, as long as the (00030) constraint is fulfilled, i.e., $\left.D_{\alpha} J_{\text {abcde }}\right|_{(00030)}$ vanishes.

We first observe that there are no corrections at order $\alpha^{\prime}$. For dimensional reasons, $F_{\alpha \beta}$ has to be proportional to $\lambda^{2}$, which does not contain the representation (00020). Then, starting at order $\alpha^{\prime 2}$, there are two types of possible terms, modulo the lowest order field equations $(A, B, \ldots$ are adjoint gauge group indices, not to be confused with $A=(a, \alpha)$ used earlier):

$$
\begin{aligned}
J_{a b c d e}^{A} & =\frac{1}{2} \alpha^{\prime 2} M_{B C D}^{A}\left(\lambda^{B} \Gamma^{f} \Gamma_{a b c d e} \Gamma^{g} \lambda^{C}\right) F_{f g}^{D} \\
& +\frac{1}{6} \alpha^{\prime 2} N^{A}{ }_{B C}\left(D_{[a} \lambda^{B} \Gamma_{b c d} D_{e]} \lambda^{C}-\text { dual }\right) .
\end{aligned}
$$

These satisfy the (00030) constraint at linear order, which is easily seen by acting with a spinor derivative and perform tensor multiplication of the representations of the fields. So far, $M$ and $N$ are kept arbitrary, but with the manifest symmetry $(B C)$. To derive the equations of motion we need some of the higher components of $J$. This will be done in a following paper [12], where the complete action at order $\alpha^{\prime 2}$ will be constructed. For the moment we will content ourselves with extracting the physically relevant deformations out of $J$. We want to determine which types of terms can be removed by fields redefinitions and which can not. The field redefinitions are taken care of by shifting $A_{\alpha}$ as explained in the previous section. These shifts can be of three independent forms at this order in $\alpha^{\prime}$, namely

$$
\begin{aligned}
\delta A_{\alpha}^{A} & =\frac{1}{6} \alpha^{\prime 2} m^{A}{ }_{B C D}\left(\lambda^{B} \Gamma^{a b c} \lambda^{C}\right)\left(\Gamma_{a b c} \lambda^{D}\right)_{\alpha} \\
& +\alpha^{\prime 2} n^{A}{ }_{B C D}\left(\lambda^{B} \Gamma^{a} \lambda^{C}\right)\left(\Gamma_{a} \lambda^{D}\right)_{\alpha} \\
& +\alpha^{\prime 2} p^{A}{ }_{B C} F^{B a b}\left(\Gamma_{a} D_{b} \lambda^{C}\right)_{\alpha} .
\end{aligned}
$$

When we calculate $\delta F_{\alpha \beta}=(\Delta \delta A)_{\alpha \beta}$, only the first and third of these contribute to (00020) (any contribution to the vector part is removed by an accompanying redefinition of $A_{a}$, so that the conventional constraint remains unaffected). The third one is used to get rid of any " $D \lambda D \lambda$ " terms in eq. (5.1), so these can be discarded as irrelevant. Examining the first (" $\lambda \lambda \lambda$ ") term in (5.2), we observe that it has mixed symmetry in the $\lambda$ 's (i.e., neither the completely symmetric nor the completely antisymmetric product of three (00001)'s contain (00010)). Consequently, $\delta J_{a b c d e}$ is proportional to $\alpha^{\prime 2} m_{B[C D]}^{A}\left(\lambda^{B} \Gamma^{f} \Gamma_{a b c d e} \Gamma^{g} \lambda^{C}\right) F_{f g}^{D}$. Since the combination of fields contracting $M$ and $m$ are manifestly symmetric in $(B C)$, this field redefinition is used to remove the part of $M$ with mixed symmetry, and the remaining relevant part of $M$ is only the one completely symmetric in $(B C D)$. For any gauge group, this seems to imply that $M$ is symmetric in all four indices. If the gauge group is $\mathrm{SU}(N)$, there are two available tensors, $d_{(A B}{ }^{E} d_{C D) E}$ and $\delta_{(A B} \delta_{C D)}$. The symmetric trace is one specific combination of these.

This discussion shows that any $F^{4}$ terms in the action (that cannot be removed by field redefinitions) are completely symmetric in the adjoint indices. This does not mean that all 
terms at this order in $\alpha^{\prime}$ are contracted with symmetric tensors; we have already seen that the field equations contain commutator terms, and we expect terms like " $F \lambda^{4}$ " contracted with an $M$ and a structure constant $f$.

It will be very interesting to examine to which degree linear supersymmetry alone determines the higher-order corrections. Clearly, once the first correction is postulated, higher ones are necessary. In our framework, we see this as the need for cancellation of the (00030) constraint at order $\alpha^{\prime 4}$. It is yet an open question to what degree there remains an arbitrariness in this procedure, i.e., if new (non-polynomial) invariants arise that start at order $\alpha^{\prime 4}$ and higher. Our intuition leads us to suspect that the higher-order interactions are not unique (we have at least evidence that an independent invariant exists that starts at or$\left.\operatorname{der} \alpha^{\prime 3}[16]\right)$, and that a second, non-linearly realised, supersymmetry is necessary in order to determine the Born-Infeld action. We envisage that the techniques of the present paper, where all the dynamics is encoded in the relatively simple object $F_{\alpha \beta}$, and where the (00030) constraint is the only condition needed to consider in the iterative procedure, is suited for addressing such questions.

We would like to compare the results from the present approach with those obtained by Bergshoeff et al. [10] by demanding a non-abelian $\kappa$-symmetry (with a spinor parameter in the adjoint of the gauge group). In that paper, quartic terms were found at order $\alpha^{\prime 2}$ that are not contracted by a completely symmetric tensor $M$. Our results seem to indicate that such terms, in any supersymmetric gauge theory, are trivial and removable by field redefinitions.

The issues and techniques discussed in this paper can be directly taken over to elevendimensional supergravity and the higher-order corrections generated by string/M-theory. In fact, one of the reasons for the study conducted here is to investigate the ideas in [17], where they were applied to M-theory, in the much simpler context of Yang-Mills theory.

Acknowledgements: We would like to thank Gabriele Ferretti for discussions and for pointing out references. This work is partly supported by EU contract HPRN-CT-2000-00122 and by the Swedish Natural Science Research Council. For some representation-theoretical considerations, such as tensor products of representations, the program LiE [18] has been very useful. 


\section{REFERENCES}

[1] A.A. Tseytlin, "Born-Infeld action, supersymmetry and string theory", in the Yuri Golfand memorial volume, ed. M. Shifman, World Scientific (2000) hep-th/9908105

[2] M. Aganagić, C. Popescu, J.H. Schwarz, "Gauge-invariant and gauge fixed D-brane actions", Nucl. Phys. B495 (1997) 145 hep-th/9612080.

[3] M. Cederwall, A. von Gussich, B.E.W. Nilsson and A. Westerberg, "The Dirichlet super-three-brane in ten-dimensional type IIB supergravity" Nucl. Phys. B490 (1997) 163 hep-th/9610148;

M. Aganagić, C. Popescu, J.H. Schwarz, "D-brane actions with local kappa symmetry", Phys. Lett. B393 (1997) 311 hep-th/9610249;

M. Cederwall, A. von Gussich, B.E.W. Nilsson, P. Sundell and A. Westerberg, "The Dirichlet super-pbranes in ten-dimensional type IIA and IIB supergravity", Nucl. Phys. B49o (1997) 179 hep-th/9611159; E. Bergshoeff and P.K. Townsend, "Super D-branes", Nucl. Phys. B49o (1997) 145 hep-th/9611173.

[4] E. Witten, "Bound states of strings and p-branes", Nucl. Phys. B46o (1996) 335 hep-th/9510135.

[5] A. Sevrin, J. Troost and W. Troost, "The non-abelian Born-Infeld action at order F" ", hep-th/0101192.

[6] P.S. Howe, O. Raetzel and E. Sezgin. "On brane actions and superembeddings". J. High Energy Phys. $\mathbf{9 8 0 8}$ (1998) o11 hep-th/9804051;

J. Bagger and A. Galperin, "A new Goldstone multiplet for partially broken supersymmetry", Phys. Rev. D55 (1997) 1091 hep-th/9608177;

T. Adawi, M. Cederwall, U. Gran, M. Holm and B.E.W. Nilsson, "Superembeddings, non-linear supersymmetry and 5-branes", Int. J. Mod. Phys. A13 (1998) 4691 hep-th/9711203;

P. Pasti, D. Sorokin and M. Tonin, "Superembeddings, partial supersymmetry breaking and superbranes", Nucl. Phys. B591 (2000) 109 hep-th/0007048].

[7] E. Bergshoeff, M. Rakowski and E. Sezgin, "Higher derivative super Yang-Mills theories, Phys. Lett. B185 (1987) 371.

[8] A.A. Tseytlin, "On the non-abelian generalization of Born-Infeld action in string theory", Nucl. Phys. B501 (1997) 41 hep-th/9701125.

[9] A. Hashimoto and W. Taylor IV, "Fluctuation spectra of tilted and intersecting D-branes from the BornInfeld action", Nucl. Phys. B503 (1997) 193 hep-th/9703217.

[10] E.A. Bergshoeff, M. de Roo and A. Sevrin, "On the supersymmetric non-abelian Born-Infeld action", hep-th/0011264.

[11] L. Brink, J.H. Schwarz and J. Scherk, "Supersymmetric Yang-Mills theories", Nucl. Phys. B121 (1977) 77 .

[12] M. Cederwall, B.E.W. Nilsson and D. Tsimpis, in preparation.

[13] B.E.W. Nilsson, "Off-shell fields for the 10-dimensional supersymmetric Yang-Mills theory", GöteborgITP-81-6; "Pure spinors as auxiliary fields in the ten-dimensional supersymmetric Yang-Mills theory", Class. Quantum Grav. 3 (1986) L41.

[14] S.J. Gates, K.S. Stelle and P.C. West, "Algebraic origins of superspace constraints in supergravity", Nucl. Phys. B169 (1980) 347; S.J. Gates and W. Siegel, "Understanding constraints in superspace formulation of supergravity", Nucl. Phys. B163 (1980) 519 .

[15] B.E.W. Nilsson, "Superspace action for a 6-dimensional non-extended supersymmetric Yang-Mills theory", Nucl. Phys. B174 (1980) 335 .

[16] Y. Kitazawa, "Effective lagrangian for open superstring from five point function", Nucl. Phys. B289 (1987) 599 .

[17] M. Cederwall, U. Gran, M. Nielsen and B.E.W. Nilsson, "Manifestly supersymmetric M-theory", J. High Energy Phys. o010 (2000) 041 hep-th/0007035|; "Generalised 11-dimensional supergravity", hepth/0010042.

[18] A.M. Cohen, M. van Leeuwen and B. Lisser, LiE v. 2.2 (1998), http://wallis.univ-poitiers.fr/ ${ }^{\sim}$ maavl/LiE/ 